\title{
Article \\ Comparison of the Chemical and Sensorial Evaluation of Dark Chocolate Bars
}

\author{
Ylenia Pieracci ${ }^{1}\left(\mathbb{D}\right.$, Roberta Ascrizzi $^{1, *} \mathbb{D}$, Luisa Pistelli ${ }^{1,2} \mathbb{D}$ and Guido Flamini ${ }^{1,2} \mathbb{D}$ \\ 1 Department of Pharmacy, University of Pisa, Via Bonanno 6, 56126 Pisa, Italy; \\ yleniapieracci@gmail.com (Y.P.); luisa.pistelli@unipi.it (L.P.); guido.flamini@unipi.it (G.F.) \\ 2 Interdepartmental Research Center "Nutraceuticals and Food for Health", University of Pisa, \\ Via del Borghetto 80, 56124 Pisa, Italy \\ * Correspondence: roberta.ascrizzi@gmail.com
}

Citation: Pieracci, Y.; Ascrizzi, R.; Pistelli, L.; Flamini, G. Comparison of the Chemical and Sensorial Evaluation of Dark Chocolate Bars. Appl. Sci. 2021, 11, 9964. https:// doi.org/10.3390/app11219964

Academic Editors: Ivelina Desseva and Dasha Mihaylova

Received: 29 September 2021

Accepted: 22 October 2021

Published: 25 October 2021

Publisher's Note: MDPI stays neutra with regard to jurisdictional claims in published maps and institutional affiliations.

Copyright: (c) 2021 by the authors. Licensee MDPI, Basel, Switzerland. This article is an open access article distributed under the terms and conditions of the Creative Commons Attribution (CC BY) license (https:// creativecommons.org/licenses/by/ $4.0 /)$.

\begin{abstract}
As it mimics olfactory perception, headspace analysis is frequently used for examination of products like chocolate, in which aroma is a key feature. Chemical analysis by itself, however, only provides half the picture, as final consumer's perception cannot be compared to that of a Gas Chromatography-Mass Spectrometry (GC-MS) port, but rather to a panel test assessment. The aim of the present study was the evaluation of combined chemical (by means of headspace solid-phase microextraction and GC-MS) and panel test data (by means of a sensory evaluation operated by 6 untrained panelists) obtained for 24 dark chocolate bars to assess whether these can discriminate between bars from different brands belonging to different commercial segments (hard discount, HD; supermarket, SM; organic bars, BIO). In all samples, with the only exception of one supermarket bar (in which esters exhibited the highest relative abundance), pyrazines were detected as the most abundant chemical class (HD: 56.3-74.2\%; BIO: 52.0-76.4\%; SM: 31.2-88.9\%). Non-terpene alcohols, aldehydes, and esters followed as quantitatively relevant groups of compounds. The obtained data was then subjected to hierarchical cluster (HCA) and principal component (PCA) analysis. The statistical distribution of samples obtained for the chemical data did not match that obtained with panelists' sensorial data. Moreover, although an overall ability of grouping samples of the same commercial origin was evidenced for hard discount and supermarket bars, no sharp grouping was possible.
\end{abstract}

Keywords: Theobroma cacao L.; cocoa; panel test; headspace; volatile organic compounds; pyrazines

\section{Introduction}

Flavor is undoubtedly the most important chocolate attribute in consumers' experience. As a complex combination of taste, aroma, and chemesthetic perceptions originating from the mouth and nasal area, many parameters concur to its development $[1,2]$. The quality of the starting raw material is of the utmost importance; cocoa varieties are, indeed, characterized by enormous genetic diversity, which ultimately confers peculiar aromatic notes to the different cultivars, classified based on their overall quality as "bulk" and "fine aroma" beans [3,4]. The cocoa market for chocolate mass production is dominated by the "bulk" cocoa, since these Theobroma cacao L. varieties are more resilient to diseases and have larger yields. However, in recent years, especially in central Europe, chocolate is more and more consumed as "gourmet" food, with higher quality standard requirements from the average consumers' market, as well [5]. In general, consumers tend to perceive cocoa beans coming from a single geographical region as an indication of higher quality of the product, but this is considered misleading by a lot of chocolate manufacturers since the terroir greatly varies within the same country, as well [5].

The subsequent manufacturing phases of the primary and secondary processing have a great influence on the quality of the final product. The flavor precursors are, indeed, developed during primary processing phases (pulp management, fermentation, 
and drying); the secondary phases (alkalization, roasting, grinding, etc.) complete the transformation of the precursors into the final flavor notes, commonly associated which chocolate [1,3]. Moreover, the processing method used determines other favorable sensory characteristics of chocolate, such as its appearance and texture, the latter perceived both on the lips and in the mouth [2].

Chocolate manufacturers and chocolatiers mainly rely on the percentage of cocoa solids to define the quality of chocolate, considering values of $75-85 \%$ as indices of a more sophisticated aroma [5]. The average consumers' preference, however, is more addressed by chocolate bars with a lower cocoa solids content, generally between $50 \%$ and $60 \%$ [6].

The analysis of volatile organic compounds (VOCs) by headspace solid phase microextraction (HS-SPME) is a fast, easy, and reliable method to assess their emission from chocolate bars of different origin. It allows identification of chemical compounds composing the aroma bouquet of the product, as perceived at room temperature as consumers' smell impression, reproducing the most similar olfactory perception conditions [7]. The starting material and the primary processing of the cocoa beans ensure the presence of aroma precursors, while the secondary processing develops the characteristic volatiles involved in chocolate aroma [1]. Ziegleder [8] identified over 600 volatile compounds involved in chocolate aroma, among which the vast majority belong to the pyrazines chemical class [9-11], followed by esters [9,12,13]. The aroma of chocolate, however, is quite complex, as it involves a wide variety of chemical groups, such as alcohols $[4,9,11,14]$, aldehydes $[9,11,15]$, and ketones [11,12,15]. Pyrones, furanones, and acids add depth to the bouquet composition, and are frequently reported as emitted volatiles, however their presence is generally perceived as pleasant only when in low relative abundances $[9,11,16]$.

The chemical assessment alone, however, does not take into account the human perception, which is far more complex since it is the result of a combination of chemical and physical stimuli. Electronic noses are widely used in food analysis, but the obtained results generally do not correlate very well with the sensorial analysis performed by a panel test, unless used to look for a specific aroma note in a matrix [17]. The complexity of the chocolate matrix, composed of a large bouquet of VOCs, each with its own aroma contribution and olfactory threshold, makes it very difficult to directly correlate the chemical profile of a product with consumers' perception. In such cases, indeed, the human sensorial analysis seems more reliable in the assessment of the overall aroma perception [7].

In the present work, the aim was the evaluation of the volatile emission profiles of dark chocolate bars by HS-SPME, coupled with GC/MS, to assess the existence of a pattern able to group them according to their commercial origin (organic, supermarket, and hard discount bars). All the bars shared a 70\% cocoa purity and did not contain milk; the cocoa percentage was chosen as a generally acceptable cocoa purity for the average consumers' preference. Moreover, a sensorial analysis was carried out by six untrained panelists, who represented the "average consumer". They analyzed a complete series of sensorial attributes based on a set of descriptors covering visual, auditory, textural, olfactive, and aroma perceptions. Finally, statistical analyses were carried out to compare the distribution of the samples according to their volatile compositions and their panel test score.

\section{Materials and Methods}

\subsection{Dark Chocolate Bars}

All the analyzed dark chocolate bars were purchased by authors in various stores (hard discounts and supermarkets) located in Pisa, Italy. The selection reflected the commercial availability of the products, since all the bars had to share the same cocoa purity (70\%) and list of ingredients (cocoa mass, cocoa butter, cocoa powder, sugar, soy or sunflower lecithin, and vanilla extract) to ensure the highest possible homogeneity among the samples.

\subsection{Headspaces Sampling}

The headspace of all the samples were analyzed by means of the headspace solid phase micro-extraction (HS-SPME) technique. All the samples (5 g each) were cut and 
individually put in $4 \mathrm{~mL}$ glass vials (up to $1 / 3$ of their total volume), which were then covered with aluminum foil and left to equilibrate at room temperature for $30 \mathrm{~min}$. A Supelco SPME (Solid Phase Micro-Extraction) device coated with polydimethylsiloxane (PDMS, $100 \mu \mathrm{m}$ ) was used to sample the headspace of the samples. SPME sampling was performed using the same new fiber, preconditioned according to the manufacturer instructions, for all the analyses. Sampling was accomplished in an air-conditioned room $\left(22 \pm 1^{\circ} \mathrm{C}\right)$ to guarantee a stable temperature. After $30 \mathrm{~min}$ of equilibration time, the fiber was exposed to the headspace for $30 \mathrm{~min}$ at room temperature. Both the equilibration and sampling times were experimentally determined to obtain an optimal adsorption of the volatiles, and to avoid both under- and over-saturation of the fiber and of the mass spectrometer ion trap. Once sampling was finished, the fiber was withdrawn into the needle and transferred to the injection port of the GC-MS system. The desorption conditions were identical for all the samples (indicated in Section 2.3). Furthermore, blanks were performed before each first SPME extraction and randomly repeated during each series. Quantitative comparisons of relative peak areas were performed between the same chemicals in the different samples.

\subsection{Gas Chromatography-Mass Spectrometry (GC-MS) Analyses and Peaks Identification}

As reported in Ascrizzi et al. [4], the GC/EI-MS analyses were performed with a Varian CP-3800 apparatus equipped with a DB-5 capillary column $(30 \mathrm{~m} \times 0.25 \mathrm{~mm}$ i.d., film thickness $0.25 \mu \mathrm{m}$ ) and a Varian Saturn 2000 ion-trap mass detector. The oven temperature was programmed rising from $60^{\circ} \mathrm{C}$ to $240{ }^{\circ} \mathrm{C}$ at $3{ }^{\circ} \mathrm{C} / \mathrm{min}$, and the injector temperature, $220^{\circ} \mathrm{C}$, transfer-line temperature, $240^{\circ} \mathrm{C}$, and carrier gas, $\mathrm{He}(1 \mathrm{~mL} / \mathrm{min})$. The acquisition parameters were as follows: full scan; scan range: $35-300 \mathrm{~m} / \mathrm{z}$; scan time: $1.0 \mathrm{sec}$; threshold: 1 count.

The identification of the constituents was based on the comparison of their retention times $\left(t_{R}\right)$ with those of pure reference samples and their linear retention indices (LRIs) determined relatively to the $t_{R}$ of a series of $n$-alkanes. The mass spectra were compared with those listed in the commercial libraries NIST 14 and ADAMS and in a home-made massspectral library, built up from MS literature $[18,19]$ combined with data experimentally obtained from pure substances and commercial essential oils of known composition.

\subsection{Panel Test}

The organoleptic chocolate bar profiles were evaluated by a panel of 6 "naive assessors" ( 3 women, 3 men), aged 20 to 35 years, selected by a panel leader. The sensory evaluation was set up before the tasting session, leading to a guided assessment (Table 1) that included all five senses, and developed merging, with slight modifications, two lists of attributes previously reported for chocolate tasting sessions $[2,20]$. A final set of descriptors was presented to the panelists to evaluate, for each sample, whether they applied or not, with a yes/no selection. The tasting was carried out in the morning, in a well-ventilated, quiet room, in a relaxed atmosphere. To avoid cross contamination, different bars were assessed in different moments of the same session by the same group of panelists. For each tasting session, each panelist was provided with 2 chocolate squares $\left(2.5 \times 2.5 \mathrm{~cm}^{2}\right)$, without any indication about the sample identity. All samples were presented to the panel test in the same size to avoid the impact of chocolate shape on sensory attributes of bars [21]. Water, apple cubes, and unflavored salty crackers were provided to the panelists between each tasting. 
Table 1. Sensory evaluation guidelines for the panel test tasting session. For each sample, the panelists had to check all that they felt applied (yes/no selection for each descriptor).

\begin{tabular}{|c|c|c|}
\hline Sense & & Descriptors \\
\hline \multirow{5}{*}{ Sight } & Color & $\begin{array}{l}\text { Light brown } \\
\text { Medium brown } \\
\text { Dark brown }\end{array}$ \\
\hline & Shininess & $\begin{array}{l}\text { Glossy } \\
\text { Matte }\end{array}$ \\
\hline & Presence of air bubbles & Yes/No \\
\hline & Presence of white spots & Yes/No \\
\hline & Presence of stripes & Yes / No \\
\hline Hearing & Snap at break & $\begin{array}{c}\text { Hard } \\
\text { Crunchy } \\
\text { Crisp } \\
\text { Light } \\
\text { Mellow } \\
\text { Muted } \\
\text { High-pitched } \\
\text { Low }\end{array}$ \\
\hline Smell & Aroma & $\begin{array}{c}\text { Flowery (aromatic notes resembling flowers) } \\
\text { Fruity (aromatic notes resembling fruits) } \\
\text { Caramel (aromatic sweet notes resembling } \\
\text { caramel, brown sugar) } \\
\text { Nutty (aromatic notes reminiscent of nut } \\
\text { products, such as hazelnuts) } \\
\text { Herbaceous ("green" aromatic notes, } \\
\text { reminiscent of cut grass) } \\
\text { Dairy (perception of "fat" notes, reminiscent of } \\
\text { butter and cheese) } \\
\text { Fermentation/oxidation (unpleasant, pungent } \\
\text { perception of fermented food) } \\
\text { Liqueur (reminiscent of alcoholic notes) } \\
\text { Plastic (unpleasant perception of "medicine-like" } \\
\text { aroma notes) } \\
\text { Animal (unpleasant perception of animal odors) }\end{array}$ \\
\hline Taste & & $\begin{array}{l}\text { Bitter } \\
\text { Sweet } \\
\text { Salty } \\
\text { Acid } \\
\text { Umami }\end{array}$ \\
\hline \multirow{4}{*}{ Touch } & Lips & $\begin{array}{l}\text { Smooth } \\
\text { Velvety } \\
\text { Grainy }\end{array}$ \\
\hline & Tongue & $\begin{array}{l}\text { Smooth } \\
\text { Velvety } \\
\text { Grainy }\end{array}$ \\
\hline & In-mouth sensation & $\begin{array}{l}\text { Astringent } \\
\text { Burning } \\
\text { Warm } \\
\text { Sparkling } \\
\text { Stinging } \\
\text { Metallic } \\
\text { Fatty } \\
\text { Oily }\end{array}$ \\
\hline & Melting & $\begin{array}{l}\text { Immediate } \\
\text { Delayed }\end{array}$ \\
\hline
\end{tabular}

\subsection{Statistical Analyses}

All statistical analyses were carried out with the JMP Pro 13.2.1 software package (SAS Institute; Cary, NC, USA). 
The hierarchical cluster analyses (HCA) were performed using Ward's method on unscaled, normalized data for both the chemical composition of sample complete headspaces and panel test evaluation responses.

Data used for the principal component analysis (PCA) of the complete headspace compositions was a $97 \times 24$ (97 individual compounds $\times 24$ samples $=2328$ data) covariance matrix. The chosen PC1 and PC2 studied $62.1 \%$ and $13.5 \%$ of the variance, respectively, for a total explained variance of $75.6 \%$.

The principal component analysis (PCA) was performed selecting the two highest principal components (PCs) obtained by the linear regressions operated on mean-centered, unscaled data; as an unsupervised method, this analysis aimed at reducing the dimensionality of the multivariate data of the matrix, whilst preserving most of the variance [22,23]. Both the HCA and the PCA methods can be applied to observe groups of samples even when there are no reference samples that can be used as a training set to establish the model.

\section{Results and Discussion}

\subsection{Headspace Compositions}

The headspace (HS) composition of all the hard discount (HD), organic (BIO), and supermarket (SM) samples are reported in Tables $2-4$, respectively.

Table 2. Complete compositions of the headspaces sampled for hard-discount chocolate bars (HD1 to HD8).

\begin{tabular}{|c|c|c|c|c|c|c|c|c|c|}
\hline \multirow[b]{2}{*}{ Compounds } & \multirow{2}{*}{ 1.r.i. ${ }^{1}$} & \multicolumn{8}{|c|}{ Relative Abundance (\%) } \\
\hline & & HD1 & HD2 & HD3 & HD4 & HD5 & HD6 & HD7 & HD8 \\
\hline ethyl acetate & 616 & -2 & - & 10.7 & 9.9 & 5.9 & 12.6 & 3.3 & 5.3 \\
\hline 2,3-butanediol & 787 & 3.3 & - & 4.8 & 2.0 & 4.8 & 1.6 & 5.9 & 1.2 \\
\hline isovaleric acid & 834 & 2.6 & - & 2.5 & 1.3 & - & - & 3.0 & 0.4 \\
\hline isopentyl acetate & 876 & - & - & 1.7 & 0.2 & - & 1.0 & 1.6 & 0.9 \\
\hline 2,6-dimethylpyrazine & 914 & - & - & 4.0 & 0.3 & - & - & - & - \\
\hline 2,5-dimethylpyrazine & 915 & 6.0 & 1.5 & - & - & - & - & 3.0 & 0.3 \\
\hline benzaldehyde & 963 & 1.9 & - & - & 1.2 & - & - & 1.3 & - \\
\hline myrcene & 990 & - & - & 0.2 & - & - & - & 0.6 & - \\
\hline n-decane & 1000 & - & - & 0.6 & - & - & - & - & - \\
\hline 2-ethyl-3-methylpyrazine & 1002 & - & - & - & - & - & - & 0.3 & - \\
\hline 2,3,5-trimethylpyrazine & 1004 & 13.4 & 5.6 & 12.1 & 6.2 & 7.1 & 9.4 & 12.9 & 5.5 \\
\hline limonene & 1031 & 1.6 & 7.2 & - & - & - & - & 0.2 & - \\
\hline (Z)- $\beta$-ocimene & 1041 & - & - & - & - & - & - & 0.2 & - \\
\hline 2-heptyl acetate & 1043 & - & 2.4 & 1.1 & 1.2 & 1.3 & 1.3 & 0.5 & 0.5 \\
\hline 2-acetylpyrrole & 1060 & 3.2 & 2.3 & 0.8 & - & - & - & - & - \\
\hline acetophenone & 1067 & - & - & - & - & 1.2 & - & 1.0 & - \\
\hline 3-ethyl-2,5-dimethylpyrazine & 1080 & 2.3 & 1.5 & - & 0.7 & 1.0 & - & 1.6 & - \\
\hline 2-ethyl-3,5-dimethylpyrazine & 1085 & - & - & 2.0 & - & - & - & - & - \\
\hline tetramethylpyrazine & 1086 & 43.7 & 47.3 & 37.1 & 65.7 & 59.9 & 62.0 & 50.8 & 58.1 \\
\hline linalool & 1100 & - & - & - & - & 0.2 & 0.7 & 0.2 & - \\
\hline nonanal & 1103 & 6.3 & 6.6 & 1.5 & 0.3 & 3.0 & - & 1.1 & - \\
\hline phenylethyl alcohol & 1110 & 3.1 & 7.4 & 1.8 & 1.5 & 4.7 & 1.9 & 1.7 & 0.7 \\
\hline 3,5-diethyl-2-methyl pyrazine & 1160 & 1.2 & 0.5 & 1.2 & 1.3 & 1.0 & - & 1.2 & 1.8 \\
\hline trans-linalool oxide (pyranoid) & 1177 & 0.1 & - & - & - & - & - & - & - \\
\hline ethyl octanoate & 1197 & - & 2.7 & - & 0.7 & 2.6 & - & 0.5 & 1.0 \\
\hline n-dodecane & 1200 & - & - & 2.4 & - & - & - & - & - \\
\hline decanal & 1205 & 0.6 & 0.8 & 0.3 & 0.2 & 0.7 & 0.7 & - & 0.4 \\
\hline $\begin{array}{l}\text { butanoic acid, 2-methyl-3-oxo-, methyl } \\
\text { ester }\end{array}$ & 1223 & 0.7 & 0.6 & 0.6 & 0.3 & 0.8 & 0.4 & 0.4 & 0.6 \\
\hline ascaridole & 1237 & - & - & - & 0.1 & - & - & 0.2 & - \\
\hline benzene acetic acid, ethyl ester & 1247 & 0.2 & - & 0.3 & 0.5 & - & - & 0.3 & 0.8 \\
\hline (Z)-anethole & 1253 & - & - & 1.0 & - & - & - & - & - \\
\hline
\end{tabular}


Table 2. Cont.

\begin{tabular}{|c|c|c|c|c|c|c|c|c|c|}
\hline \multirow{2}{*}{ Compounds } & \multirow{2}{*}{ 1.r.i. 1} & \multicolumn{8}{|c|}{ Relative Abundance (\%) } \\
\hline & & HD1 & HD2 & HD3 & HD4 & HD5 & HD6 & HD7 & HD8 \\
\hline 2-phenylethylacetate & 1258 & 3.4 & 2.9 & 2.1 & 4.6 & 3.6 & 4.2 & 2.6 & 12.2 \\
\hline nonanoic acid & 1273 & 2.5 & 3.6 & - & - & - & 1.1 & 0.2 & 0.3 \\
\hline (E)-anethole & 1287 & - & - & 6.2 & 1.1 & - & - & 1.2 & - \\
\hline 2-undecanone & 1294 & - & - & - & 0.1 & - & 0.4 & - & - \\
\hline n-tridecane & 1300 & - & - & - & - & 0.6 & - & 0.2 & - \\
\hline undecanal & 1305 & - & - & - & 0.2 & - & - & - & - \\
\hline$\delta$-elemene & 1340 & - & - & - & - & - & - & 0.1 & - \\
\hline$\alpha$-terpinyl acetate & 1351 & - & - & - & - & - & 0.3 & - & - \\
\hline$\alpha$-copaene & 1376 & 0.6 & - & - & - & - & - & 0.2 & - \\
\hline$\beta$-patchoulene & 1380 & - & - & - & - & - & - & - & 2.3 \\
\hline ethyl decanoate & 1396 & - & - & - & - & 0.5 & - & - & - \\
\hline n-tetradecane & 1400 & - & - & 1.3 & - & 0.6 & - & - & - \\
\hline longifolene & 1403 & - & - & - & - & - & - & 0.2 & - \\
\hline methyl eugenol & 1404 & - & - & - & - & - & 0.3 & - & - \\
\hline (Z)-caryophyllene & 1405 & - & - & - & - & - & - & 0.2 & - \\
\hline$\beta$-duprezianene & 1423 & - & - & - & - & - & - & - & 1.1 \\
\hline (E)-geranyl acetone & 1454 & 1.0 & 1.4 & 0.5 & 0.3 & 0.4 & 0.8 & 0.3 & 2.6 \\
\hline$\alpha$-patchoulene & 1456 & - & - & - & - & - & - & 0.2 & - \\
\hline alloaromadendrene & 1461 & - & - & - & - & - & 0.5 & - & - \\
\hline drima-7,9(11)-diene & 1470 & - & - & - & - & - & - & - & 1.3 \\
\hline 1-dodecanol & 1471 & - & 3.2 & - & - & - & - & - & - \\
\hline trans-cadina-1(6),4-diene & 1475 & - & - & - & - & - & - & - & 1.9 \\
\hline germacrene D & 1480 & - & - & - & - & - & - & 0.5 & - \\
\hline cis- $\beta$-guaiene & 1489 & - & - & - & - & - & - & 0.2 & - \\
\hline bicyclogermacrene & 1495 & - & - & - & - & - & 0.5 & - & - \\
\hline trans- $\beta$-guaiene & 1501 & 0.4 & - & 0.6 & - & - & 0.5 & 0.5 & - \\
\hline trans- $\gamma$-cadinene & 1513 & - & - & - & - & - & - & 0.4 & - \\
\hline$\delta$-cadinene & 1523 & - & - & 0.6 & - & - & - & 0.5 & - \\
\hline ethyl dodecanoate & 1595 & - & - & 0.2 & 0.1 & - & - & 0.2 & 0.8 \\
\hline n-hexadecane & 1600 & - & - & 0.2 & - & - & - & - & - \\
\hline citronellyl pentanoate & 1625 & - & - & 0.8 & - & - & - & - & - \\
\hline cadalene & 1675 & 1.9 & 2.5 & 1.0 & - & - & - & 0.4 & - \\
\hline Monoterpene hydrocarbons & & 1.6 & 7.2 & 0.2 & - & - & - & 1.0 & - \\
\hline Oxygenated monoterpenes & & 0.1 & - & 0.8 & 0.1 & 0.2 & 1.0 & 0.4 & - \\
\hline Sesquiterpene hydrocarbons & & 2.9 & 2.5 & 2.2 & - & - & 1.5 & 3.4 & 6.7 \\
\hline Pyrazines & & 66.6 & 56.4 & 56.3 & 74.2 & 69.0 & 71.3 & 69.9 & 65.6 \\
\hline Pyrroles & & 3.2 & 2.3 & 0.8 & - & - & - & - & - \\
\hline Apocarotenoids & & 1.0 & 1.4 & 0.5 & 0.3 & 0.4 & 1.1 & 0.3 & 2.6 \\
\hline Phenylpropanoids & & - & - & 7.2 & 1.1 & - & - & 1.2 & - \\
\hline Non-terpene acids & & 5.2 & 3.6 & 2.5 & 1.3 & - & 1.1 & 3.2 & 0.8 \\
\hline Non-terpene alcohols & & 6.4 & 10.6 & 6.6 & 3.6 & 9.6 & 3.4 & 7.5 & 1.9 \\
\hline Non-terpene aldehydes & & 8.8 & 7.4 & 1.9 & 1.8 & 3.7 & 0.7 & 2.4 & 0.4 \\
\hline Non-terpene esters & & 4.3 & 8.5 & 16.6 & 17.5 & 14.7 & 19.5 & 9.4 & 22.1 \\
\hline Non-terpene ketones & & - & - & - & 0.1 & 1.2 & 0.4 & 1.0 & - \\
\hline Non-terpene hydrocarbons & & - & - & 4.4 & - & 1.2 & - & 0.2 & - \\
\hline Total identified (\%) & & 100.0 & 99.9 & 100.0 & 99.9 & 100.0 & 100.0 & 99.9 & 100.0 \\
\hline
\end{tabular}

${ }^{1}$ Linear retention indices on a DB5 capillary column. ${ }^{2}$ Not detected. Legend of the sample names: the HD code stands for hard discount, followed by a unique number, each identifying one specific commercial sample among this type. 
Table 3. Complete compositions of the headspaces sampled for organic dark chocolate bars (BIO1 and BIO2).

\begin{tabular}{|c|c|c|c|}
\hline \multirow{2}{*}{ Compounds } & \multirow{2}{*}{ 1.r.i. ${ }^{1}$} & \multicolumn{2}{|c|}{ Relative Abundance (\%) } \\
\hline & & BIO1 & $\mathrm{BIO} 2$ \\
\hline ethyl acetate & 616 & 22.3 & -2 \\
\hline 2,3-butanediol & 787 & 1.1 & 2.2 \\
\hline isovaleric acid & 834 & 2.6 & 2.1 \\
\hline isopentyl acetate & 876 & 1.0 & 1.0 \\
\hline 2,6-dimethylpyrazine & 914 & - & 2.9 \\
\hline 2,5-dimethylpyrazine & 915 & 0.9 & - \\
\hline benzaldehyde & 963 & 0.6 & - \\
\hline myrcene & 990 & - & 0.4 \\
\hline 2,3,5-trimethylpyrazine & 1004 & 5.9 & 16.5 \\
\hline limonene & 1031 & 0.1 & 2.6 \\
\hline 2-heptyl acetate & 1043 & 2.0 & 1.1 \\
\hline acetophenone & 1067 & 0.6 & 1.0 \\
\hline trans-linalool oxide (furanoid) & 1076 & 1.6 & - \\
\hline 3-ethyl-2,5-dimethylpyrazine & 1080 & 0.2 & 3.0 \\
\hline tetramethylpyrazine & 1086 & 44.3 & 51.5 \\
\hline trans-sabinene hydrate & 1099 & - & 2.6 \\
\hline linalool & 1100 & - & 0.4 \\
\hline nonanal & 1103 & 1.1 & - \\
\hline isopentyl isovalerate & 1105 & 0.8 & - \\
\hline phenylethyl alcohol & 1110 & 3.0 & 0.5 \\
\hline 3,5-diethyl-2-methyl pyrazine & 1160 & 0.8 & 2.5 \\
\hline cis-pinocarveol & 1184 & 0.1 & - \\
\hline ethyl octanoate & 1197 & 0.7 & 0.7 \\
\hline decanal & 1205 & 0.4 & 0.3 \\
\hline $\begin{array}{c}\text { butanoic acid, } \\
\text { 2-methyl-3-oxo-, methyl ester }\end{array}$ & 1223 & 0.4 & 0.5 \\
\hline ascaridole & 1237 & 0.2 & - \\
\hline benzene acetic acid, ethyl ester & 1247 & 0.8 & - \\
\hline 2-phenylethylacetate & 1258 & 4.7 & 5.5 \\
\hline nonanoic acid & 1273 & 0.9 & - \\
\hline (E)-anethole & 1287 & - & 2.5 \\
\hline$n$-tridecane & 1300 & 0.6 & - \\
\hline undecanal & 1305 & 0.4 & - \\
\hline n-tetradecane & 1400 & 0.4 & - \\
\hline dodecanal & 1408 & 0.2 & - \\
\hline (E)-geranyl acetone & 1454 & 0.7 & 0.4 \\
\hline$n$-pentadecane & 1500 & 0.2 & - \\
\hline $\begin{array}{l}\text { benzoic acid, 4-ethoxy-, ethyl } \\
\text { ester }\end{array}$ & 1522 & 0.4 & - \\
\hline \multicolumn{2}{|c|}{ Monoterpene hydrocarbons } & 0.1 & 3.0 \\
\hline \multicolumn{2}{|c|}{ Oxygenated monoterpenes } & 2.0 & 2.9 \\
\hline \multicolumn{2}{|c|}{ Pyrazines } & 52.0 & 76.4 \\
\hline \multicolumn{2}{|c|}{ Apocarotenoids } & 0.7 & 0.4 \\
\hline \multicolumn{2}{|c|}{ Phenylpropanoids } & - & 2.5 \\
\hline \multicolumn{2}{|c|}{ Non-terpene acids } & 3.5 & 2.1 \\
\hline \multicolumn{2}{|c|}{ Non-terpene alcohols } & 4.2 & 2.7 \\
\hline \multicolumn{2}{|c|}{ Non-terpene aldehydes } & 2.5 & 0.3 \\
\hline \multicolumn{2}{|c|}{ Non-terpene esters } & 33.3 & 8.8 \\
\hline \multicolumn{2}{|c|}{ Non-terpene ketones } & 0.6 & 1.0 \\
\hline \multicolumn{2}{|c|}{ Non-terpene hydrocarbons } & 1.2 & - \\
\hline \multicolumn{2}{|c|}{ Total identified (\%) } & 100.0 & 100.0 \\
\hline
\end{tabular}

${ }^{1}$ Linear retention indices on a DB5 capillary column. ${ }^{2}$ Not detected. Legend of the sample names: the BIO code stands for organic, followed by a unique number, each identifying one specific commercial sample among this type. 
Table 4. Complete compositions of the headspaces sampled for supermarket chocolate bars (SM1 to SM14).

\begin{tabular}{|c|c|c|c|c|c|c|c|c|c|c|c|c|c|c|c|}
\hline \multirow{2}{*}{ Compounds } & \multirow[b]{2}{*}{ 1.r.i. ${ }^{1}$} & \multicolumn{14}{|c|}{ Relative Abundance (\%) } \\
\hline & & SM1 & SM2 & SM3 & SM4 & SM5 & SM6 & SM7 & SM8 & SM9 & SM10 & SM11 & SM12 & SM13 & SM14 \\
\hline ethyl acetate & 616 & -2 & 0.1 & - & - & - & - & - & - & - & 0.2 & - & 6.7 & 20.1 & 3.8 \\
\hline pentanal & 695 & - & - & - & - & - & - & - & - & - & - & - & 13.5 & - & 7.0 \\
\hline 2,3-butanediol & 787 & 4.4 & 2.2 & 2.0 & - & - & - & 2.1 & - & - & 0.4 & 1.6 & 5.5 & - & 5.9 \\
\hline isovaleric acid & 834 & 4.1 & 2.5 & 4.1 & 3.1 & - & - & 2.6 & 1.2 & 1.1 & 0.7 & 0.9 & 5.4 & - & 3.7 \\
\hline isopentyl acetate & 876 & - & - & 1.9 & - & - & 0.5 & - & 4.5 & - & 0.2 & 0.5 & 9.4 & - & 6.7 \\
\hline 2,6-dimethylpyrazine & 914 & 3.9 & 1.0 & - & - & - & - & 2.6 & - & 1.2 & - & - & - & - & - \\
\hline 2,5-dimethylpyrazine & 915 & - & - & 3.1 & 1.6 & 3.6 & - & - & - & - & 0.7 & 0.6 & 3.2 & - & 3.6 \\
\hline ethyl acetoacetate & 940 & - & 8.7 & - & - & - & - & - & - & - & - & - & - & - & - \\
\hline benzaldehyde & 963 & 1.1 & - & 2.4 & - & - & 0.3 & 1.5 & - & 0.2 & 0.2 & - & 0.3 & - & 0.1 \\
\hline myrcene & 990 & - & - & 1.4 & - & - & 0.2 & 1.9 & - & 0.9 & - & - & 0.6 & - & 0.3 \\
\hline 2-ethyl-6-methylpyrazine & 994 & - & - & 0.6 & - & - & - & - & - & - & - & - & - & - & 0.3 \\
\hline$m$-mentha-1(7),8-diene & 1001 & - & - & - & - & - & - & - & - & - & - & - & 0.4 & - & - \\
\hline 2,3,5-trimethylpyrazine & 1004 & 9.2 & 7.2 & 9.6 & 4.8 & 13.1 & 9.1 & 9.9 & 5.1 & 7.9 & 8.6 & 6.5 & 8.1 & 3.7 & 14.7 \\
\hline limonene & 1031 & 0.5 & - & 0.3 & - & - & - & - & - & - & - & - & - & 3.2 & - \\
\hline sylvestrene & 1032 & 0.2 & - & - & - & - & - & - & - & - & - & - & - & - & - \\
\hline 1,8-cineole & 1035 & - & 0.4 & - & - & 1.7 & - & 1.0 & - & - & - & - & - & - & - \\
\hline$(Z)$ - $\beta$-ocimene & 1041 & - & - & - & - & - & - & 0.3 & - & - & - & - & 0.2 & - & - \\
\hline 2-heptyl acetate & 1043 & 2.0 & - & - & 0.5 & - & 0.8 & - & - & 0.8 & - & - & 1.8 & - & 0.7 \\
\hline 2-acetylpyrrole & 1060 & 1.8 & - & 4.7 & 1.1 & 2.3 & - & 2.9 & 1.6 & 1.7 & - & - & 0.9 & - & 0.8 \\
\hline acetophenone & 1067 & - & 0.8 & - & - & - & - & - & - & - & - & 0.4 & - & - & - \\
\hline trans-linalool oxide (furanoid) & 1076 & - & 1.3 & - & - & - & - & - & - & - & 0.7 & - & - & - & - \\
\hline 3-ethyl-2,5-dimethylpyrazine & 1080 & 1.1 & - & - & 0.4 & 2.7 & 1.0 & 1.8 & 0.5 & 1.0 & 0.8 & 0.6 & - & - & 1.5 \\
\hline 2,6-diethylpyrazine & 1081 & - & - & 0.9 & - & - & - & - & - & - & - & - & - & - & - \\
\hline 2-ethyl-3,5-dimethylpyrazine & 1085 & - & - & - & - & - & - & - & - & - & - & - & 0.9 & - & - \\
\hline tetramethylpyrazine & 1086 & 56.3 & 59.7 & 51.2 & 24.4 & 56.5 & 70.7 & 55.5 & 69.1 & 66.7 & 77.0 & 75.1 & 34.2 & 27.8 & 45.2 \\
\hline linalool & 1100 & 2.4 & - & - & - & - & - & - & - & 3.4 & - & - & 1.9 & - & - \\
\hline nonanal & 1103 & - & 2.3 & 2.9 & 1.3 & 3.4 & 0.6 & 3.5 & 3.0 & - & - & - & - & 8.0 & - \\
\hline phenylethyl alcohol & 1110 & 4.0 & 3.8 & 2.7 & 2.8 & 3.4 & 3.6 & 2.3 & 2.3 & 1.4 & 1.3 & 2.0 & 0.6 & 7.6 & 0.8 \\
\hline 3,5-diethyl-2-methyl pyrazine & 1160 & 0.7 & 1.3 & 0.5 & - & 1.1 & 0.6 & 1.0 & 1.3 & 1.3 & 1.8 & 1.2 & 0.7 & - & 1.6 \\
\hline ethyl benzoate & 1173 & 0.2 & - & 0.2 & - & - & 0.3 & - & - & - & - & - & - & - & - \\
\hline trans-linalool oxide (pyranoid) & 1177 & - & - & - & - & - & - & - & 0.1 & 0.3 & - & - & - & 0.4 & - \\
\hline butanedioic acid, diethyl ester & 1185 & 0.2 & - & - & - & - & - & - & - & - & - & - & - & - & - \\
\hline 1-dodecene & 1192 & - & - & - & - & 0.6 & - & - & - & - & - & - & - & - & - \\
\hline ethyl octanoate & 1197 & 1.2 & 0.8 & 0.4 & 0.3 & - & 0.7 & 0.8 & 0.6 & 0.8 & 1.0 & 0.6 & 0.6 & 1.6 & 0.6 \\
\hline n-dodecane & 1200 & - & - & - & - & 1.8 & - & - & - & - & - & - & - & - & - \\
\hline decanal & 1205 & 0.6 & 0.6 & 0.7 & 0.3 & 1.3 & - & 0.4 & 0.3 & 0.2 & 0.2 & 0.2 & - & 1.5 & - \\
\hline $\begin{array}{c}\text { butanoic acid, 2-methyl-3-oxo-, methyl } \\
\text { ester }\end{array}$ & 1223 & 0.8 & 0.6 & 0.6 & 0.4 & 0.7 & - & 0.6 & 0.6 & 0.6 & 0.5 & - & 0.5 & 1.3 & 0.4 \\
\hline ascaridole & 1237 & - & - & 0.2 & - & - & - & 0.3 & 0.1 & 0.3 & - & - & 0.2 & - & - \\
\hline benzene acetic acid, ethyl ester & 1247 & - & - & 0.4 & - & - & - & - & - & 0.5 & - & 0.7 & 0.2 & 1.7 & 0.2 \\
\hline (Z)-anethole & 1253 & - & - & - & - & - & - & - & - & - & - & - & 1.2 & - & - \\
\hline 2-phenylethylacetate & 1258 & 3.9 & 6.1 & 4.5 & 1.6 & 2.0 & 7.2 & 5.2 & 5.5 & 5.6 & 4.0 & 5.8 & 2.3 & 11.1 & 1.6 \\
\hline nonanoic acid & 1273 & - & - & 0.8 & - & 0.4 & 0.6 & 1.8 & - & 1.2 & - & - & - & - & - \\
\hline (E)-anethole & 1287 & - & - & - & - & - & - & - & - & - & 0.9 & 2.6 & 0.3 & 6.9 & 0.3 \\
\hline$n$-tridecane & 1300 & - & - & - & - & 0.6 & - & 0.3 & - & 0.1 & - & - & - & - & - \\
\hline undecanal & 1305 & - & - & - & - & - & - & - & - & - & - & - & - & 0.3 & - \\
\hline undec-9-en-1-al & 1315 & - & - & - & - & - & - & 0.2 & - & - & - & - & - & - & - \\
\hline dihydrocitronellol acetate & 1321 & - & - & - & - & 0.5 & - & - & - & - & - & - & - & - & - \\
\hline$\alpha$-copaene & 1376 & - & - & - & - & - & - & 0.3 & 0.1 & 0.2 & - & - & - & 0.5 & - \\
\hline$\beta$-patchoulene & 1380 & - & - & - & - & - & - & - & - & - & - & - & - & 0.2 & - \\
\hline$\beta$-panasinsene & 1383 & - & - & 0.2 & - & - & - & - & - & - & - & - & - & - & - \\
\hline vanillin & 1394 & - & - & - & - & - & - & 0.3 & 3.1 & 1.4 & - & - & - & - & - \\
\hline sativene & 1395 & - & - & 0.3 & - & - & - & - & - & - & - & - & - & - & - \\
\hline ethyl decanoate & 1396 & - & - & - & - & - & 0.6 & - & - & - & 0.4 & 0.3 & - & - & - \\
\hline n-tetradecane & 1400 & 0.5 & - & 0.5 & - & 2.1 & - & - & - & - & - & - & 0.2 & - & - \\
\hline longifolene & 1403 & - & - & 0.1 & - & - & - & - & - & - & - & - & - & - & - \\
\hline dodecanal & 1408 & 0.3 & - & - & - & 0.6 & - & - & - & - & - & - & - & - & - \\
\hline$\beta$-ylangene & 1414 & - & - & - & - & - & - & - & - & - & - & - & - & 0.4 & - \\
\hline (E)-caryophyllene & 1418 & 0.2 & 0.2 & - & - & - & - & - & - & - & - & - & - & - & - \\
\hline (trans,cis)-iridolactone & 1446 & - & - & - & 6.3 & - & - & - & - & - & - & - & - & - & - \\
\hline$(E)$-geranyl acetone & 1454 & 0.4 & 0.2 & 1.8 & 4.7 & 1.0 & - & 0.2 & 0.4 & 0.3 & - & 0.2 & - & 2.1 & - \\
\hline (E)-ethyl cinnamate & 1462 & - & - & - & - & - & - & - & 0.1 & 0.2 & - & - & - & - & - \\
\hline (E)-2-dodecen-1-ol & 1465 & - & - & - & 0.7 & - & - & - & - & - & - & - & - & - & - \\
\hline$\gamma$-muurolene & 1477 & - & - & - & - & - & - & 0.3 & - & - & - & - & - & - & - \\
\hline n-pentadecane & 1500 & - & - & - & - & - & - & 0.2 & - & - & - & - & - & - & - \\
\hline$(Z)-\alpha$-bisabolene & 1503 & - & - & - & - & 0.3 & - & - & - & - & - & - & - & - & - \\
\hline benzoic acid, 4-ethoxy-, ethyl ester & 1522 & - & - & 0.8 & - & - & - & - & - & - & - & - & - & - & - \\
\hline$\delta$-cadinene & 1523 & - & - & - & - & - & - & - & - & - & 0.1 & - & - & 0.6 & - \\
\hline trans-cadinene ether & 1559 & - & - & - & - & - & - & - & - & 0.3 & - & - & - & - & - \\
\hline (E)-dehydroapofarnesol & 1591 & - & - & - & 19.4 & - & - & - & - & - & - & - & - & - & - \\
\hline ethyl dodecanoate & 1595 & - & - & - & - & - & - & 0.5 & 0.3 & 0.4 & 0.2 & 0.2 & 0.1 & 1.0 & 0.2 \\
\hline epi-cedrol & 1598 & - & - & - & 9.4 & - & - & - & - & - & - & - & - & - & - \\
\hline$n$-hexadecane & 1600 & - & - & - & - & - & 2.5 & - & - & - & - & - & - & - & - \\
\hline tetradecanal & 1612 & - & - & - & 16.5 & - & - & - & - & - & - & - & - & - & - \\
\hline cadalene & 1675 & - & - & - & - & - & - & - & - & - & - & 0.2 & - & - & 0.2 \\
\hline tetradecanol & 1676 & - & 0.4 & - & - & - & - & - & 0.1 & - & - & - & - & - & - \\
\hline
\end{tabular}


Table 4. Cont.

\begin{tabular}{|c|c|c|c|c|c|c|c|c|c|c|c|c|c|c|c|}
\hline \multirow{2}{*}{ Compounds } & \multirow{2}{*}{ l.r.i. ${ }^{1}$} & \multicolumn{14}{|c|}{ Relative Abundance (\%) } \\
\hline & & SM1 & SM2 & SM3 & SM4 & SM5 & SM6 & SM7 & SM8 & SM9 & SM10 & SM11 & SM12 & SM13 & SM14 \\
\hline Monoterpene hydrocarbons & & 0.7 & - & 1.7 & - & - & 0.2 & 2.2 & - & 0.9 & - & - & 1.2 & 3.2 & 0.3 \\
\hline Oxygenated monoterpenes & & 2.4 & 1.7 & 0.2 & - & 2.1 & - & 1.3 & 0.3 & 4.0 & 0.7 & - & 2.1 & 0.4 & - \\
\hline Sesquiterpene hydrocarbons & & 0.2 & 0.2 & 0.6 & - & 0.3 & - & 0.6 & 0.1 & 0.2 & 0.1 & 0.2 & - & 1.8 & 0.2 \\
\hline Oxygenated sesquiterpenes & & - & - & - & 28.8 & - & - & - & - & 0.3 & - & - & - & - & - \\
\hline Pyrazines & & 71.2 & 69.1 & 65.9 & 31.2 & 77.0 & 81.4 & 70.7 & 76.0 & 78.1 & 88.9 & 83.9 & 47.1 & 31.5 & 67.0 \\
\hline Pyrroles & & 1.8 & - & 4.7 & 1.1 & 2.3 & - & 2.9 & 1.6 & 1.7 & - & - & 0.9 & - & 0.8 \\
\hline Apocarotenoids & & 0.4 & 0.2 & 1.8 & 4.7 & 1.0 & - & 0.2 & 0.4 & 0.3 & - & 0.2 & 0.0 & 2.1 & - \\
\hline Phenylpropanoids & & - & - & - & - & - & - & - & 0.1 & 0.2 & 0.9 & 2.6 & 1.6 & 6.9 & 0.3 \\
\hline Non-terpene acids & & 4.1 & 2.5 & 4.9 & 3.1 & 0.4 & 0.6 & 4.4 & 1.2 & 2.4 & 0.7 & 0.9 & 5.4 & - & 3.7 \\
\hline Non-terpene alcohols & & 8.5 & 6.3 & 4.8 & 3.4 & 3.4 & 3.6 & 4.4 & 2.4 & 1.4 & 1.8 & 3.6 & 6.2 & 7.6 & 6.7 \\
\hline Non-terpene aldehydes & & 2.0 & 2.9 & 6.0 & 18.1 & 5.3 & 0.8 & 5.9 & 6.4 & 1.8 & 0.4 & 0.2 & 13.8 & 9.7 & 7.2 \\
\hline Non-terpene esters & & 8.3 & 16.3 & 8.8 & 2.8 & 2.6 & 10.0 & 7.1 & 11.6 & 8.7 & 6.6 & 8.0 & 21.6 & 36.8 & 13.9 \\
\hline Non-terpene ketones & & 0.0 & 0.8 & - & 6.3 & - & - & - & - & - & - & 0.4 & - & - & - \\
\hline Non-terpene hydrocarbons & & 0.5 & - & 0.5 & - & 5.0 & 2.5 & 0.5 & - & 0.1 & - & - & 0.2 & - & - \\
\hline Total identified (\%) & & 100.0 & 100.0 & 99.9 & 99.5 & 99.4 & 99.1 & 100.0 & 100.0 & 100.0 & 100.0 & 100.0 & 100.0 & 100.0 & 100.0 \\
\hline
\end{tabular}

${ }^{1}$ Linear retention indices on a DB5 capillary column. ${ }^{2}$ Not detected. Legend of the sample names: the SM code stands for supermarket, followed by a unique number, each identifying one specific commercial sample among this type.

Pyrazines were the most represented chemical class of volatile organic compounds (VOCs) in all HD the samples, as they ranged from a minimum of $56.3 \%$ in HD3 to a maximum of $74.2 \%$ in HD4. Pyrazines were detected as the most abundant VOC chemical class in BIO samples, as well, where they accounted for $52.0 \%$ and $76.4 \%$ in $\mathrm{BIO} 1$ and $\mathrm{BIO} 2$, respectively. With the only exception of sample SM13, pyrazines were detected as the most abundant chemical class of VOCs in all SM samples, too, accounting for up to almost $90 \%$ in SM10. In SM4 headspace (HS), however, their relative content was only slightly higher $(31.2 \%)$ than that of oxygenated sesquiterpenes (28.8\%). Among this chemical class, tetramethylpyrazine and 2,3,5-trimethylpyrazine were the most quantitatively relevant in all samples. Pyrazine are heterocyclic volatiles produced during the Maillard reaction, whose aroma contribution is of the utmost importance in chocolate, as they are responsible for its typical pleasant flavor $[1,24,25]$. Tetramethylpyrazine was found as the most abundant compound in all HSs, with relative abundances always higher than $35 \%$. Its odor is described as reminiscent of coffee, with a green and roasted aroma [2,12,15]. 2,3,5-Trimethylpyrazine followed; its aroma contribution is similar to that of tetramethylpyrazine, but with a slightly earthier and roasted-nuts like flavor $[15,26,27]$.

Non-terpene esters followed as the second most abundant VOC chemical class in HD3 to HD8 samples, with relative abundances varying between $9.4 \%$ and $22.1 \%$ in HD7 and HD8, respectively. Their quantitative importance was also evidenced for BIO (33.3\% in $\mathrm{BIO} 1,8.8 \%$ in $\mathrm{BIO} 2$ ) and $\mathrm{SM}$ (from $2.6 \%$ in SM5, up to $36.8 \%$ in SM13) samples, although with great variation based on the bar brand. Among them, ethyl acetate and 2-phenylethyl acetate were the most represented. Pyrazines decrement in SM13 and BIO1 were coupled with the increment of non-terpene esters, in particular of that of ethyl acetate. Ethyl and acetate esters are indeed reported as important VOCs involved in chocolate aroma [12,13]. In SM4, instead, their decrement was coupled with an increment in sesquiterpene hydrocarbons, which confer green and spicy notes to the product [28].

Among HD bars, HD1 HS demonstrated the highest relative content of non-terpene aldehydes, of which nonanal was the most quantitatively important. These compounds also exhibited a quantitatively relevant presence in SM4 and SM12, as they reached up to $18.1 \%$ and $13.8 \%$, respectively; among them, the former was mainly rich in tetradecanal, while pentanal reached $13.5 \%$ in the latter. In BIO bars, they were detected with a $2.5 \%$ relative abundance in $\mathrm{BIO} 1$, while in $\mathrm{BIO} 2$ they only accounted for $0.3 \%$. Fruity and flowery notes are associated to aliphatic volatile aldehydes [15], among which the most abundant detected in the present study were nonanal and pentanal. The former is associated with citrus-like aroma notes [29], while the latter is more pungent and bitter [1]. A citrus peellike flavor is also described for tetradecanal [29], which was only detected in sample SM4 but with a significant relative abundance. In this study, benzaldehyde followed among the 
most detected aldehydes, whose highest relative concentration was registered in sample HD1; it is listed among the undesirable notes, as it is pungent and bitter [11,12,14,15].

Non-terpene alcohols, another typical chemical class of VOCs found in chocolate headspaces, were identified in relevant relative concentrations in all samples (HD: 1.9-10.6\%; BIO: 2.7-4.2\%; SM: 1.4-8.5\%). Among them, phenylethylalcohol and 2,3-butanediol were the most represented. Non-terpene alcohol presence is commonly reported as a desirable attribute of chocolate aroma, as they are responsible for pleasant flowery $[1,4,9,11]$ and sweet [11] notes. Among those detected in the present study, 2,3-butanediol, whose highest relative abundances were identified in samples SM12 and 14, and HD3, 5, and 7, confer cocoa butter aroma notes $[4,14,30]$. Phenylethyl alcohol is reported as a pleasant odor in chocolate samples, reminiscent of honey [11]: its highest relative quantities were found in samples SM13 and HD2. Dodecanol, whose presence was detected only in sample HD2, is described as a delicate floral note when diluted, but it turns into an unpleasant aroma when present in significant content [29].

Among non-terpene compounds, acids were also detected in almost all samples (HD: 0.8-5.2\%; BIO: 2.1-3.5\%; SM: 0.4-5.4\%), with only two exceptions (HD5 and SM13). Of this class, nonanoic and isovaleric acid were the two most represented in all HSs.

(E)-Anethole was the most represented detected phenylopropanoid compound, varying between samples in which it was not detected at all, to headspaces in which its presence was quantitatively relevant (6.2\% in HD3; $6.9 \%$ in SM13).

In HD HSs, relative terpene concentrations over $5 \%$ were only found for HD2 and HD8; in the former, monoterpene hydrocarbons, only represented by limonene, were up to $7.2 \%$, while in the latter sesquiterpene hydrocarbons prevailed, with $\beta$-patchoulene as the most represented. Among terpenes, (E)-dehydroapofarnesol and epi-cedrol, two oxygenated monoterpenes undetected in all the other SM samples, were detected in relative abundances up to $19.4 \%$ and $9.4 \%$, respectively, in SM4.

Unsurprisingly, all the samples demonstrated volatile emissions mainly composed of pyrazines, with tetramethylpyrazine as the main compound in all HSs. Moreover, esters, especially acetates, followed as expected, being the second most reported chemical group of volatile compounds commonly reported in chocolate products. The other compounds demonstrated an overall higher degree of variation between the samples, especially in quantitative terms. The typical aroma of a chocolate product is, indeed, the result of a complex interaction between the starting material quality and the following processing phases, which add the "matrix-effect" to the release dynamics of the very same volatile compounds, thus conferring a unique aroma bouquet for each bar.

\subsection{Statistical Analysis of Chemical Data}

The hierarchical cluster analysis (HCA) dendrogram is reported in Figure 1.

Two main macro-groups were identified by the HCA; the first one (red group, Figure 1) mainly composed of SM bars, and a larger, more varied one composed of two sub-groups (green and blue, Figure 1). Although no completely sharp distribution was evidenced among the samples, an overall grouping of bars sharing the same commercial origin was visible for SM and HD samples. BIO bars were not clustered very close; this could be due to the high variability induced by the raw starting material and processing method, as well as to their smaller sample size. 


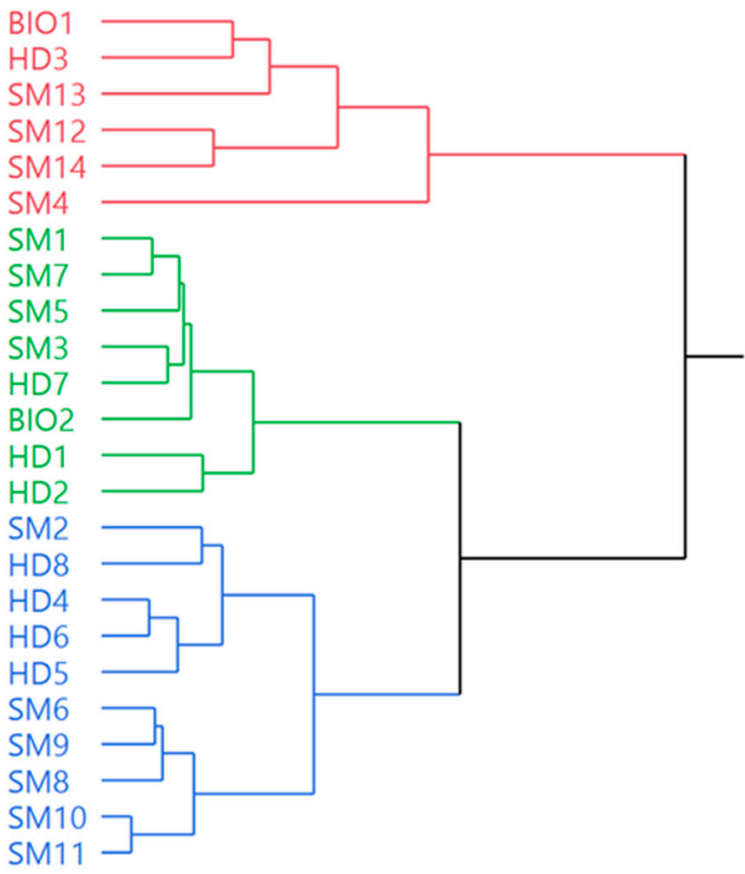

Figure 1. Dendrogram of the hierarchical cluster analysis (HCA) performed on the complete headspace compositions of all bars. Legend for the sample names: organic bars (BIO), supermarket bars (SM), and hard discount bars (HD).

The score and loading plots of the principal component analysis (PCA) are reported in Figure 2.
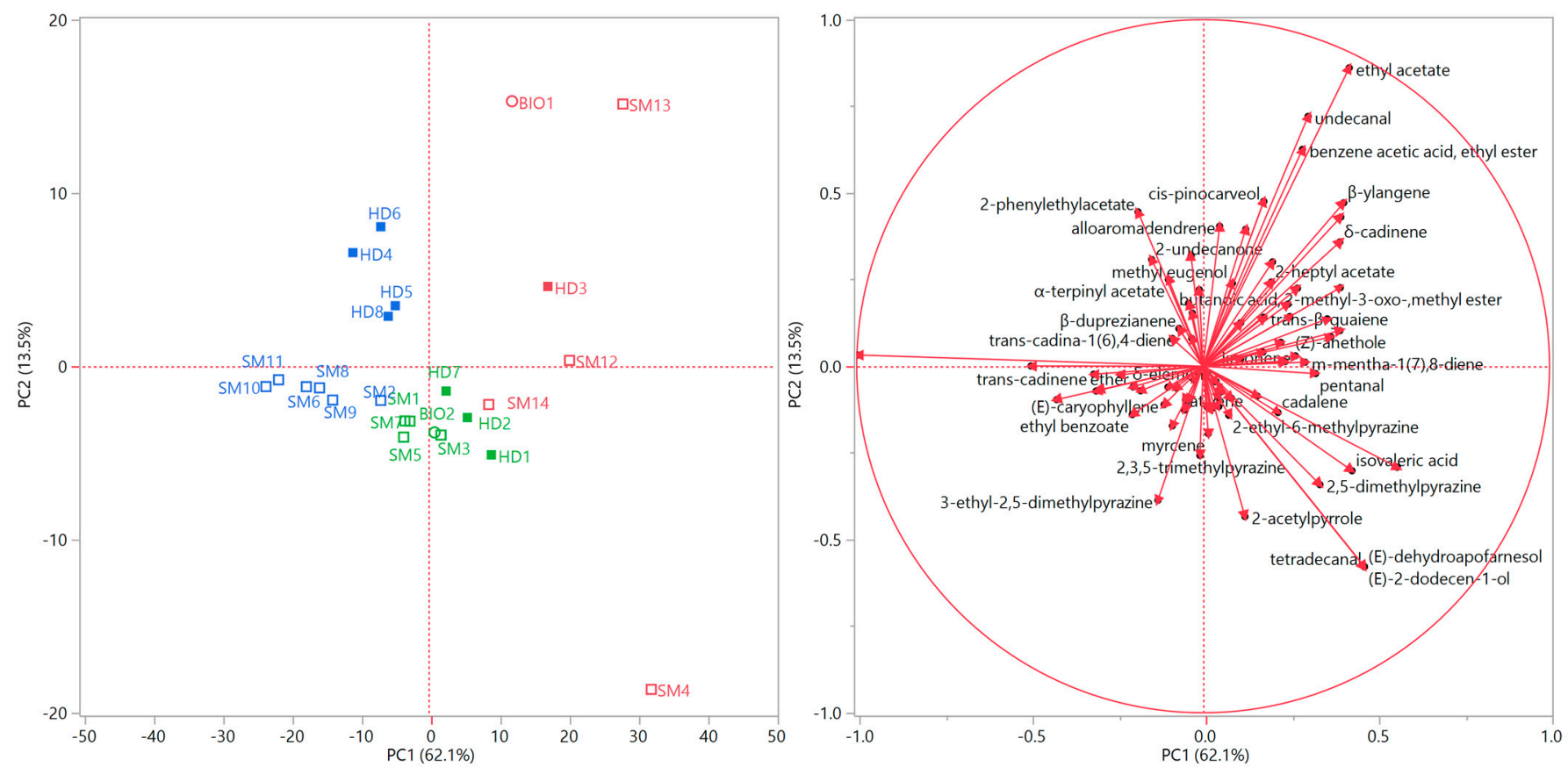

Figure 2. Score (left) and loading (right) plots of the principal component analysis (PCA) performed on the complete headspace compositions of all bars. Legend for the sample names: organic bars (BIO), supermarket bars (SM), and hard discount bars (HD).

Half of HD bars (HD4, 5, 6, and 8) were plotted in the upper left quadrant $(\mathrm{PC} 1<0$, PC2 $>0$ ) of the PCA score plot (Figure 2, left); this positioning was due to their higher content in tetramethyl pyrazine, whose vector was sharply directed towards the 
left side of the loadings plot (Figure 2, right). The other $4 \mathrm{HD}$ bars were all distributed in the right quadrants $(\mathrm{PC} 1>0)$, between the bottom area of the upper quadrant $(\mathrm{PC} 2>0)$ and the upper area of the bottom quadrant $(\mathrm{PC} 2<0)$; compared to the other HD bars, their compositions were richer in nonanal, whose vector pointed towards the right quadrant of the loadings plot (Figure 2, right). Samples previously grouped by HCA in the first macro-cluster (samples in red, Figure 1) were all plotted in the right $(\mathrm{PC} 1>0)$ score plot quadrants, where only a few samples of the other macro-cluster were positioned, in the bottom right quadrant $(\mathrm{PC} 2<0)$. All other samples from the second HCA macro-cluster were grouped quite close to each other towards the central area of the left quadrants $(\mathrm{PC} 1<0)$, mainly due to tetramethylpyrazine vector (Figure 2, right).

Samples plotted quite distant from all others were BIO1 and SM13 in the upper right quadrant (PC1 and PC2 $>0$ ), due to their higher relative content in ethyl acetate (Figure 2, right), and SM4 in the bottom right quadrant (PC1 $>0, P C 2<0)$, whose position was due to the tetradecanal vector (Figure 2, right).

The VOCs emission, used as a tool to evidence the overall distribution of samples by statistical means, could not sharply divide samples based on their commercial origin; however, it demonstrated a general ability to group SM and HD bars. The 2 BIO samples, however, were clustered and plotted as two very distant samples; a larger difference in degree in the raw material quality and/or the processing technology might thus be hypothesized for these samples compared to their SM and HD counterparts. However, the size of the BIO group is too small to draw definitive conclusions on the matter. In accordance with the results emerged from the HSs analysis, each bar, no matter its commercial fragment of origin (HD, SM, or BIO), displayed a unique profile, based on all the phases involved in the development of an aroma bouquet as complex as that of chocolate. The overall aroma profiles for all samples were, indeed, composed of the expected main chemical groups (pyrazines and esters). The secondary chemical classes, such as aldehydes, ketones, and alcohols, displayed higher variability among the samples, but without evidencing any pattern that might be attributed to the commercial segment of the product. This is quite interesting, as the commercial origin of chocolate might influence consumers' preference towards bars perceived as having a higher quality (i.e., oganic and supermarket bars over the hard discount ones.).

\subsection{Panel Test}

The average score for each descriptor as perceived by the six untrained panelists for all the samples is reported in Table 5. Each descriptor had a yes/no response; thus, the scores in Table 5 were calculated by assessing how many panelists had attributed each descriptor for the bars of the same commercial origin (hard discount, HD; organic, BIO; super market, $\mathrm{SM}$ ), averaging it based on the number of bars for each provenience ( $8 \mathrm{HD}$, $2 \mathrm{BIO}, 14 \mathrm{SM})$.

The brown color intensity resulted higher for HD and SM bars, while BIO bars where perceived as lighter in color. HD bars also displayed the highest gloss rate, as well as the highest number of samples with air bubbles, while BIO and SM bars demonstrated the highest average scores for the presence of stripes ("sugar bloom" effect) on bar surfaces. HD bars scored the highest glossiness rate, while $\mathrm{SM}$ and $\mathrm{BIO}$ bars appearance was described as matte.

The appearance of chocolate is an important hedonic parameter in determining consumers' preference. The color intensity is mainly determined by the temper regime; under-tempered chocolate, in particular, develops a lighter color as fat blooming creates fat crystals which scatter the light, resulting in a paler appearance [31]. Among the analyzed chocolates, organic bars exhibited a lighter shade (medium brown) compared to the dark brown descripted by panelists in HD and SM bars. However, the oily descriptor for the in-mouth perception scored higher in the HD bars. The gloss parameter is also influenced by the tempering process, together with the particle size; a higher fat blooming degree in under-tempered chocolates, as well as an increase in the particle size, reduce the desirable 
glossy appearance of the final product [31]. Among visual markers of poor chocolate production, HD bars exhibited the highest number of samples with air bubbles, indicating that the molds in which the tempered chocolate was poured were too cold [32]. The BIO and SM bars, instead, scored higher average values for surface stripes, signs of the "sugar bloom" effect, which occurs when either pods are stored in too humid places or an intermediate product is too rapidly transferred from low to high temperatures; this ultimately causes the water to reach the surface, where it dissolves sugar and then evaporates, leaving a white appearance due to the remaining sugar crystals [33].

Table 5. Average scores of the panel test (6 untrained panelists) evaluation descriptors for the organic (BIO, 2 samples), hard discount (HD, 8 samples), and supermarket (SM, 14 samples) bars.

\begin{tabular}{|c|c|c|c|}
\hline Descriptors & BIO & HD & SM \\
\hline \multicolumn{4}{|c|}{ Visual features } \\
\hline Light brown & 0.0 & 0.4 & 0.4 \\
\hline Medium brown & 4.0 & 1.8 & 2.2 \\
\hline Dark brown & 2.0 & 3.8 & 3.4 \\
\hline Shiny & 2.5 & 4.0 & 2.2 \\
\hline Matte & 3.5 & 2.0 & 3.8 \\
\hline Presence of air bubbles & 0.0 & 2.0 & 0.7 \\
\hline Absence of air bubbles & 6.0 & 4.0 & 5.3 \\
\hline Presence of white spots & 1.5 & 1.3 & 1.6 \\
\hline Absence of white spots & 4.5 & 4.8 & 4.4 \\
\hline Presence of stripes & 2.5 & 1.6 & 2.1 \\
\hline Absence of stripes & 3.5 & 4.4 & 3.9 \\
\hline \multicolumn{4}{|c|}{ Snap sound at breakage } \\
\hline Hard & 2.0 & 0.9 & 2.0 \\
\hline Crunchy & 2.5 & 0.6 & 1.1 \\
\hline Crisp & 0.5 & 0.6 & 0.5 \\
\hline Light & 2.5 & 1.6 & 1.8 \\
\hline Mellow & 1.0 & 1.8 & 0.6 \\
\hline Muted & 0.0 & 0.6 & 0.1 \\
\hline High-pitched & 0.0 & 0.8 & 1.0 \\
\hline Low & 0.0 & 1.0 & 1.0 \\
\hline \multicolumn{4}{|c|}{ Odor attributes } \\
\hline Flowery & 0.0 & 0.9 & 0.9 \\
\hline Fruity & 2.5 & 2.0 & 1.8 \\
\hline Caramel & 2.0 & 0.8 & 1.3 \\
\hline Nutty & 1.5 & 0.6 & 1.1 \\
\hline Herbaceous & 1.0 & 1.0 & 0.8 \\
\hline Dairy & 0.0 & 0.3 & 0.1 \\
\hline Fermentation & 0.0 & 0.0 & 0.3 \\
\hline Malty & 0.0 & 0.0 & 0.1 \\
\hline Animal aroma & 0.0 & 0.4 & 0.0 \\
\hline \multicolumn{4}{|c|}{ Aroma attributes } \\
\hline Flowery & 0.0 & 0.3 & 0.6 \\
\hline Fruity & 1.5 & 1.5 & 1.5 \\
\hline Caramel & 0.5 & 1.4 & 1.2 \\
\hline Nutty & 2.0 & 1.3 & 1.1 \\
\hline Herbaceous & 1.5 & 0.8 & 0.9 \\
\hline Dairy & 0.0 & 0.4 & 0.3 \\
\hline Fermentation & 0.5 & 0.4 & 0.7 \\
\hline Malty & 0.0 & 0.0 & 0.1 \\
\hline Plastic & 0.0 & 0.0 & 0.1 \\
\hline Animal & 0.0 & 0.6 & 0.1 \\
\hline
\end{tabular}


Table 5. Cont.

\begin{tabular}{|c|c|c|c|}
\hline Descriptors & BIO & HD & SM \\
\hline \multicolumn{4}{|c|}{ Texture and melting attributes } \\
\hline Smooth (lips) & 4.0 & 4.1 & 3.6 \\
\hline Velvety (lips) & 1.5 & 1.6 & 1.9 \\
\hline Grainy (lips) & 0.5 & 0.5 & 0.5 \\
\hline Smooth (tongue) & 2.5 & 2.8 & 2.4 \\
\hline Velvety (tongue) & 3.0 & 3.3 & 2.9 \\
\hline Grainy (tongue) & 0.5 & 0.1 & 0.6 \\
\hline Immediate melting & 1.0 & 2.6 & 1.1 \\
\hline Delayed melting & 5.0 & 3.4 & 4.9 \\
\hline \multicolumn{4}{|c|}{ Taste perception } \\
\hline Bitter taste & 5.0 & 3.4 & 3.5 \\
\hline Sweet taste & 2.0 & 2.8 & 2.4 \\
\hline Salty taste & 0.5 & 0.8 & 0.4 \\
\hline Acidic taste & 0.0 & 1.1 & 1.6 \\
\hline Umami taste & 0.0 & 0.4 & 0.2 \\
\hline \multicolumn{4}{|c|}{ In-mouth sensation } \\
\hline Astringent sensation & 2.0 & 0.9 & 0.9 \\
\hline Burnt sensation & 0.0 & 0.1 & 0.1 \\
\hline Warmth sensation & 1.5 & 1.6 & 1.7 \\
\hline Sparkling sensation & 0.5 & 0.4 & 0.8 \\
\hline Acrid sensation & 0.0 & 0.3 & 0.4 \\
\hline Metallic sensation & 0.0 & 0.1 & 0.2 \\
\hline Fatty sensation & 0.0 & 0.6 & 0.4 \\
\hline Oily sensation & 2.0 & 1.6 & 1.1 \\
\hline
\end{tabular}

Legend for the sample names: organic bars (BIO), supermarket bars (SM), and hard discount bars (HD).

Organic and supermarket bars were reported as having harder and crunchier snap sounds, while lighter and more mellow sounds were described for the HD bars. The lighter and more mellow sound perceived by the panelists when breaking the HD bars compared to the harder notes reported for the organic and supermarket bars might indicate a higher presence of fats, and especially in their unsaturation degree, since this parameter positively correlated with more unpleasant softness and lack of snap in the final product [34]; on the contrary, a good, high-pitched snap at ambient conditions is a desirable character in chocolate [2].

The reported odor attributes were mainly fruity for all bars; in BIO and SM bars the caramel-like odor followed, while for HD bars the herbaceous odor was the second overall most attributed descriptor. The aroma, perceived in the retro-nasal area once bars were put in the mouth, was quite consistent with their odor perception; high scores for the fruity aroma notes were described for all bars, but BIO bars scored higher in the nutty descriptor, while the caramel-like contribution was attributed more to HD and SM bars. Both odor and aroma negative attributes of dairy and animal-like notes were overall more perceived in HD bars. Fruity notes perceived in all bars can be attributed to the quantitatively relevant presence, in all samples, of 2-phenylethyl acetate, whose aroma contribution is reported as pleasantly sweet and fruity $[1,11,12]$. The more intense herbaceous odor perceived by panelists in $\mathrm{HD}$ bars could be ascribed to their overall higher relative content of 2,5-dimethylpyrazine, whose olfactory contribution is described as a "green" note [4,35]. HD bars were also richer in nonanal, a non-terpene aldehyde to which their higher scores in the dairy and animal-like aroma and odor notes can be attributed, since its contribution is reported as "fatty" [29]. In both odor and aroma perception, the nutty descriptor was mainly attributed to the HD bars; this might be due to the overall higher relative content of benzaldehyde in HD bars [36]. Aldehydes, in general, are released faster during mastication, as they have less interaction with the oral mucosa [37]. 
The texture of the chocolate matrix is a result of its particles distribution and size; solid particles over $35 \mu \mathrm{m}$ are, indeed, detected by the tongue during mastication, causing a rough and gritty in-mouth perception [2]. Most of the chocolate bars were perceived as smooth on the lips; the textural perception then turned velvety once the product reached the tongue, due to the fat melting triggered by the higher in-mouth temperature. Moreover, the majority of the bars exhibited a delayed in-mouth melting.

The bitter taste attribute prevailed in panelists' descriptions of most of the bars, especially BIO samples. The sweet descriptor followed, and it was mainly scored by HD bars. The in-mouth sensation after mastication is an after-taste perception which persists even after the swallowing. The astringency perceived by the panelists when tasting the $\mathrm{BIO}$ bars is a sharp and, to a certain degree, pleasant sensation, related to the origin of the used cocoa $[2,4,38]$; however, if it is too strong and turns to sour, it might be an indication of over-fermentation of the beans [2].

The two in-mouth sensations most attributed to BIO bars were astringent and oily; for HD and SM bars the warm and oily in-mouth sensations prevailed.

Overall, the HD bars scored the highest number of negative characteristics, whose presence might be attributed to the use of a higher fat content. Their appearance was, indeed, glossier and the in-mouth oily feeling was higher in these bars, which let us hypothesize that the tempering phase was conducted with higher fat concentrations. This was also confirmed by lighter and more mellow snap sound and by the higher scores of perceived negative aroma attributes (dairy and animal notes). The BIO bars were more characterized by a higher degree of acidic notes and, thus, astringency, and also by a lighter color, which might indicate those bars were under-tempered. SM bars displayed intermediate characteristics, with an overall predominance of a sweet taste and fruity aroma.

\subsection{Statistical Analysis of Panel Test Data}

The dendrogram of the hierarchical cluster analysis (HCA) performed on the panel test data is reported in Figure 3.

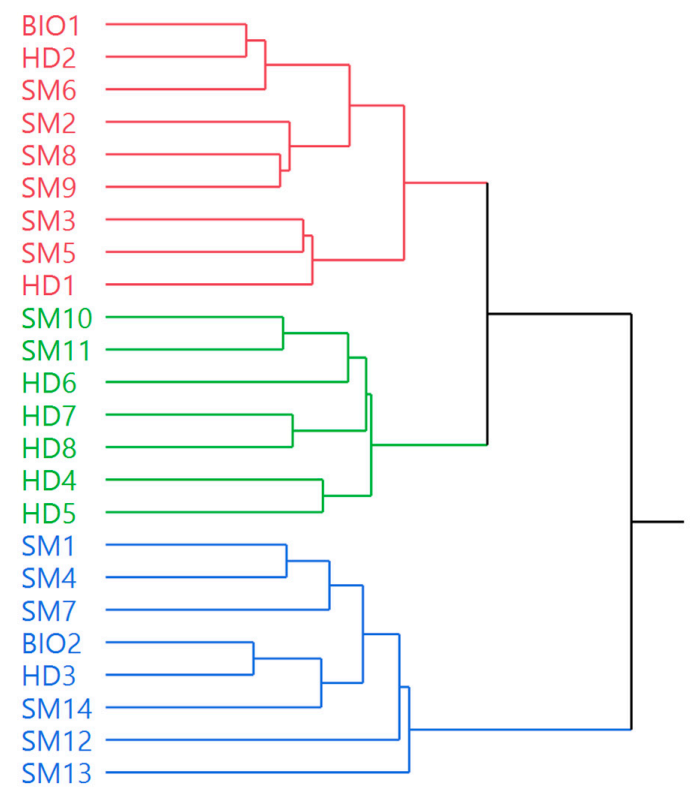

Figure 3. Dendrogram of the hierarchical cluster analysis (HCA) performed on the panel test data for all samples. Legend for the sample names: organic bars (BIO), supermarket bars (SM), and hard discount bars (HD).

Two macro-groups were distinguished by the HCA; the first one comprising two sub-groups (red and green samples, Figure 3), and the second one comprising only one 
group of bars (blue samples, Figure 3). Although no completely sharp classification of the bars based on their commercial origin was evidenced by the panel test evaluation, SM and HD bars were, overall, grouped close to similar origin samples. For BIO bars, as previously referred for the chemical analysis, the low sample number might have played a role in their completely different clustering, as evidenced in the HCA performed on the chemical composition of their headspaces, as well. As evidenced in for the chemical data, the statistical analyses performed on the panel test results could not provide a strict distribution of samples based on their commercial origin, although a general grouping of SM and HD samples was obtained.

\section{Conclusions}

Chemical data obtained by HS-SPME analysis of chocolate bars represent valuable tools to evaluate the emitted odor-active compounds; among these, unpleasant odors are especially useful to assess evident flaws due to the raw starting material and/or the production process. Although a general ability of these data to group, by statistical means, samples based on their commercial origin was evidenced for both SM and HD bars, the clustering and the plotting were not able to sharply define bars provenience.

Neither panel test results, whose evaluation includes more parameters, however, were able to clearly separate chocolate bars based on their commercial origin; moreover, samples distribution provided by hierarchical cluster analysis performed on these data did not match with that obtained when evaluating the VOC emission.

Chemical and panel test data evidenced that the commercial origin of a chocolate bar sample does not provide a certain key to assess the quality of the product, although they appeared to point out a low degree of variability among HD and SM samples.

Author Contributions: Conceptualization, G.F.; methodology, G.F. and R.A.; software, R.A. and Y.P.; validation, G.F. and R.A.; formal analysis, Y.P.; investigation, R.A.; resources, L.P. and G.F.; data curation, R.A. and Y.P.; writing-original draft preparation, R.A.; writing-review and editing, G.F., Y.P. and L.P.; visualization, R.A.; supervision, G.F. and L.P.; project administration, L.P. and G.F. All authors have read and agreed to the published version of the manuscript.

Funding: This research received no external funding.

Institutional Review Board Statement: Not applicable.

Informed Consent Statement: Not applicable.

Conflicts of Interest: The authors declare no conflict of interest.

\section{References}

1. Ascrizzi, R.; Pistelli, L.; Flamini, G. Aroma Evolution in Chocolate Production. In Food Aroma Evolution; Bordiga, M., Nollet, L., Eds.; CRC Press: Boca Raton, FL, USA, 2020; pp. 383-409.

2. Afoakwa, E.O. Sensory character and flavour perception of chocolates. In Chocolate Science and Technology; Wiley-Blackwell: Chichester, UK, 2016; pp. 202-222.

3. Qin, X.-W.; Lai, J.-X.; Tan, L.-H.; Hao, C.-Y.; Li, F.-P.; He, S.-Z.; Song, Y.-H. Characterization of volatile compounds in Criollo, Forastero and Trinitario cocoa seeds (Theobroma cacao L.) in China. Int. J. Food Prop. 2016, 2912, 2261-2275. [CrossRef]

4. Ascrizzi, R.; Flamini, G.; Tessieri, C.; Pistelli, L. From the raw seed to chocolate: Volatile profile of Blanco de Criollo in different phases of the processing chain. Microchem. J. 2017, 133, 474-479. [CrossRef]

5. Cidell, J.L.; Alberts, H.C. Constructing quality: The multinational histories of chocolate. Geoforum 2006, 37, 999-1007. [CrossRef]

6. Januszewska, R.; Viaene, J. Acceptance of chocolate by preference cluster mapping across Belgium and Poland. J. Euromarketing 2002, 11, 61-86. [CrossRef]

7. Lozano, J.; Santos, J.; Arroyo, T.; Aznar, M.; Cabellos, J.; Gil, M.; Horrillo, M. Correlating e-nose responses to wine sensorial descriptors and gas chromatography-mass spectrometry profiles using partial least squares regression analysis. Sens. Actuators $B$ Chem. 2007, 127, 267-276. [CrossRef]

8. Ziegleder, G. Linalool contents as characteristic of some flavor grade cocoas. Z. Für Leb. Und. Forsch. 1990, 191, 306-309. [CrossRef]

9. Aprotosoaie, A.C.; Luca, S.V.; Miron, A. Flavor Chemistry of Cocoa and Cocoa Products-An Overview. Compr. Rev. Food Sci. Food Saf. 2016, 15, 73-91. [CrossRef] 
10. Afoakwa, E.O.; Paterson, A.; Fowler, M.; Ryan, A. Flavor Formation and Character in Cocoa and Chocolate: A Critical Review. Crit. Rev. Food Sci. Nutr. 2008, 48, 840-857. [CrossRef]

11. Rodriguez-Campos, J.; Escalona-Buendía, H.B.B.; Contreras-Ramos, S.M.M.; Orozco-Avila, I.; Jaramillo-Flores, E.; Lugo-Cervantes, E. Effect of fermentation time and drying temperature on volatile compounds in cocoa. Food Chem. 2012, 132, 277-288. [CrossRef]

12. Rodriguez-Campos, J.; Escalona-Buendia, H.B.; Orozco-Avila, I.; Lugo-Cervantes, E.; Jaramillo-Flores, M.E. Dynamics of volatile and non-volatile compounds in cocoa (Theobroma cacao L.) during fermentation and drying processes using principal components analysis. Food Res. Int. 2011, 44, 250-258. [CrossRef]

13. Ramli, N.; Hassan, O.; Said, M.; Samsudin, W.; Idris, N. Influence of roasting conditions on volatile flavor of roasted Malaysian cocoa beans. J. Food Process. Preserv. 2006, 30, 280-298. [CrossRef]

14. Ramos, C.L.; Dias, D.R.; Miguel, M.G.d.C.P.; Schwan, R.F. Impact of different cocoa hybrids (Theobroma cacao L.) and S. cerevisiae UFLA CA11 inoculation on microbial communities and volatile compounds of cocoa fermentation. Food Res. Int. 2014, 64, 908-918. [CrossRef] [PubMed]

15. Bonvehí, J.S. Investigation of aromatic compounds in roasted cocoa powder. Eur. Food Res. Technol. 2005, 221, 19-29. [CrossRef]

16. Krings, U.; Berger, R.G.; Banavara, D.S. Thin layer high vacuum distillation to isolate the flavor of high-fat food. Eur. Food Res. Technol. 2003, 217, 70-73. [CrossRef]

17. Di Natale, C.; Macagnano, A.; Paolesse, R.; Mantini, A.; Tarizzo, E.; D’Amico, A.; Sinesio, F.; Bucarelli, F.; Moneta, E.; Quaglia, G. Electronic nose and sensorial analysis: Comparison of performances in selected cases. Sens. Actuators B Chem. 1998, 50, $246-252$. [CrossRef]

18. Adams, R.P. Identification of Essential Oil Components by Gas Chromatography/Mass Spectroscopy; Allured Publishing Corporation: Carol Stream, IL, USA, 2007; ISBN 1932633219.

19. National Institute of Standards and Technology. NIST/EPA/NIH Mass Spectral Library; The NIST Mass Spectrometry Data Center: Gaithersburg, MD, USA, 2014.

20. Yuh, E. The Chocolate Tasting Guide; Chronicle Books LLC: San Francisco, CA, USA, 2014.

21. Lenfant, F.; Hartmann, C.; Watzke, B.; Breton, O.; Loret, C.; Martin, N. Impact of the shape on sensory properties of individual dark chocolate pieces. LWT Food Sci. Technol. 2013, 51, 545-552. [CrossRef]

22. Choi, Y.H.; Kim, H.K.; Hazekamp, A.; Erkelens, C.; Lefeber, A.W.M.; Verpoorte, R. Metabolomic Differentiation of Cannabis sativa Cultivars Using 1H NMR Spectroscopy and Principal Component Analysis. J. Nat. Prod. 2004, 67, 953-957. [CrossRef]

23. Ascrizzi, R.; Flamini, G.; Giusiani, M.; Stefanelli, F.; Deriu, V.; Chericoni, S. VOCs as fingerprints for the chemical profiling of hashish samples analyzed by HS-SPME/GC-MS and multivariate statistical tools. Forensic. Toxicol. 2018, 36, 243-260. [CrossRef]

24. Beckett, S.T. The Science of Chocolate, 2nd ed.; Royal Society of Chemistry: Cambridge, UK, 2015; ISBN 9781788012355.

25. Yu, A.N.; Zhang, A.D. The effect of $\mathrm{pH}$ on the formation of aroma compounds produced by heating a model system containing 1-ascorbic acid with 1-threonine/l-serine. Food Chem. 2010, 119, 214-219. [CrossRef]

26. Frauendorfer, F.; Schieberle, P. Changes in key aroma compounds of Criollo cocoa beans during roasting. J. Agric. Food Chem. 2008, 56, 10244-10251. [CrossRef]

27. Frauendorfer, F.; Schieberle, P. Identification of the key aroma compounds in cocoa powder based on molecular sensory correlations. J. Agric. Food Chem. 2006, 54, 5521-5529. [CrossRef] [PubMed]

28. Slaghenaufi, D.; Ugliano, M. Norisoprenoids, Sesquiterpenes and Terpenoids Content of Valpolicella Wines During Aging: Investigating Aroma Potential in Relationship to Evolution of Tobacco and Balsamic Aroma in Aged Wine. Front. Chem. 2018, 6, 66. [CrossRef] [PubMed]

29. Burdock, G.A. Fenaroli's Handbook of Flavor Ingredients, 6th ed.; CRC Press: Boca Raton, FL, USA, 2010; ISBN 978-1-4200-9077-2.

30. Schwan, R.F.; Wheals, A.E. The microbiology of cocoa fermentation and its role in chocolate quality. Crit. Rev. Food Sci. Nutr. 2004, 44, 205-221. [CrossRef]

31. Afoakwa, E.O.; Paterson, A.; Fowler, M.; Vieira, J. Effects of tempering and fat crystallisation behaviour on microstructure, mechanical properties and appearance in dark chocolate systems. J. Food Eng. 2008, 89, 128-136. [CrossRef]

32. Afoakwa, E.O. Chocolate Manufacturing and Processing Technology. In Cocoa Production and Processing Technology; CRC Press: Boca Raton, FL, USA, 2014; pp. 183-194.

33. Afoakwa, E.O. Chocolate Quality and Defects. In Cocoa Production and Processing Technology; CRC Press: Boca Raton, FL, USA, 2014; pp. 197-204.

34. Hendrickx, H.; De Moor, H.; Huyghebaert, A.; Janssen, G. Manufacture of chocolate containing hydrogenated butterfat. Rev. Int. La Choc. 1971, 26, 186-197.

35. Afoakwa, E.O. The chemistry of flavour development during cocoa processing and chocolate manufacture. In Chocolate Science and Technology; Wiley-Blackwell Publishers: Chichester, UK, 2016; pp. 154-170.

36. Owusu, M.; Petersen, M.A.; Heimdal, H. Relationship of sensory and instrumental aroma measurements of dark chocolate as influenced by fermentation method, roasting and conching conditions. J. Food Sci. Technol. 2013, 50, 909-917. [CrossRef] [PubMed]

37. Charles, M.; Romano, A.; Yener, S.; Barnabà, M.; Navarini, L.; Märk, T.D.; Biasoli, F.; Gasperi, F. Understanding flavour perception of espresso coffee by the combination of a dynamic sensory method and in-vivo nosespace analysis. Food Res. Int. 2015, 69, 9-20. [CrossRef]

38. Acierno, V.; Liu, N.; Alewijn, M.; Stieger, M.; van Ruth, S.M. Which cocoa bean traits persist when eating chocolate? Real-time nosespace analysis by PTR-QiToF-MS. Talanta 2019, 195, 676-682. [CrossRef] [PubMed] 